\title{
CYANOPHYCEAN FLORA OF SOUTHERN HIMANCHAL PRADESH, INDIA
}

\author{
R.K. Dwivedi, S.K. Shukla, C.P. Shukla, P.K. Misra and M.K. Seth* \\ Phycology Research Laboratory, Department of Botany \\ University of Lucknow, Lucknow 226007, India \\ *Department of Bio-sciences \\ Himanchal Pradesh University, Shimla 171003, India \\ Email:misrapkm@yahoo.com
}

\begin{abstract}
The present paper deals with 37 taxa, 18 genera, 32 species, 4 varieties and 1 forma explored from southern Himanchal Pradesh of India. These taxa are represented by Microcystis, Chroococcus, Aphanocapsa, Aphanothece, Merismopedia, Coelosphaerium, Gomphosphaeria, Stichosiphon, Oscillatoria, Lyngbya, Pseudanabaena, Cylindrospermum, Anabaena, Nostoc, Nodularia, Fortiea, Calothrix, Anabaenopsis. Out of 37 taxa, 27 are first report from the study area.
\end{abstract}

Key words: Fresh-water, blue-green algae, systematics, southern Himanchal Pradesh, India.

\section{INTRODUCTION}

Earlier works of Singh $(1959,1961)$, Talpasayi (1962), Kamat (1968, 1973), Gupta and Nair (1962), Darbal et al. (1978), Khan (1970), Habib (1999), and Seth et al. (2006), on freshwater algae of western Himalaya of India, reveal that a large part of this region is still unexplored. Habib et al. (1998) have reported chlorococcales from Kotdwar. Habib and Chaturvedi (1999, 2000, 2001) have described chlorococcales and chaetophorales from Ramnagar, Ranikhet and Kumaon region, respectively. Shukla and Misra (2006), and Shukla et al. (2007) have worked on fresh-water algae from foothills of western Himalayas. Misra et al. (2006) have reported some desmids form Himanchal Pradesh (western Himalayas).

\section{MATERIALS AND METHODS}

Random sampling technique has been applied in the algal collection procedure. Sample collections were made between November 2003 to November 2006 from lotic and lentic habitats of southern Himanchal Pradesh, India, by using planktonic mesh net. The algal samples were preserved in $3-4 \%$ formalin and slides were prepared by staining cyanophycean algae with methylene blue and mounted in glycerine. Detailed studies were made by examining specimens under a Nikon Labophot microscope E-400 with H-III photomicrographic attachment. All measuring scales given for algal photographs are equal to 10 $\mu \mathrm{m}$ except some which are equal to $50 \mu \mathrm{m}$ and are mentioned above the scale.

Species identifications have been done after Desikachary (1959), Prescott (1951) and Prasad and Srivastava (1992). 


\section{RESULTS AND DISCUSSION}

A Total of 37 taxa, 18 genera, 32 species, 4 varieties and 1 forma belonging to class Cyanophyceae have been recorded. Aphanocapsa and Fortiea have been reported only from Una district, while genus Stichosiphon, Anabaenopsis from Hamirpur district, Fortiea and Pseudanabaena from Mandi district and Cylindrocapsa from Sirmaur district only. Genus Oscillatoria Vaucher has been present at every region. Twenty seven taxa are being reported for the first time from this study area that are represented with an asterisk mark $(*)$ in explanation of figures (Plates 1 and 2)

Order: Chroococcales

Family: Chroococcaceae

Genus: Microcystis Kütz.

1. Microcystis aeruginosa Kutz. (P1. 1, Fig. 1) Desikachary, T.V. (1959) (P1. 17, Figs. 1, 2, 6, PP. 93)

Collection Number and Date: HP/MAN/6b, $21 / 5 / 2004$

Locality: Rewalsar Lake, Mandi.

2. M. flos-aquae (Wittr.) Kirchner (P1. 1, Fig. 2) Desikachary, T.V. (1959) (Pl. 17, Fig. 11, PP. 94)

Collection Number and Date: HP/MAN/6b, $21 / 5 / 2004$

Locality: Rewalsar Lake, Mandi.

Description: Cells 3-4 $\mu \mathrm{m}$ in diameter.

3. M. lamelliformis Holsinger (P1. 1, Fig. 4) Desikachary, T.V. (1959) (Pl. 19, Figs. 1, 2, PP. 91)

Collection Number and Date: HP/HAM/5c, 2/6/2005

Locality: Taal Pond, Hamirpur.

Description: Cells 3-4 $\mu \mathrm{m}$ in diameter.
4. M. robusta (Clark) Nygaard (Pl. 1, Fig. 3) Desikachary, T.V. (1959) (Pl. 17, Fig. 7-10, PP. 85)

Collection Number and Date: $\mathrm{HP} / \mathrm{HAM} / \mathrm{Sc}$, 2/6/2005

Locality: Taal Pond, Hamirpur.

Description: Cells 6-8 $\mu \mathrm{m}$ in diameter.

Genus: Chroococcus Nag.

1. Chroococcus limneticus Lemm. (Pl. 1, Fig. 5) Desikachary, T.V. (1959) (Pl. 26, Fig. 4-6, PP. 107)

Collection Number and Date: HP/UNA/4c, $1 / 6 / 2005$

Locality: Swan River Una.

Description: Cells without sheath $10 \mu \mathrm{m}$ in diameter with sheath $14 \mu \mathrm{m}$ in diameter.

2. C. minutus (Kutz.) Nag. (Pl. 1, Fig. 7) Desikachary, T.V. (1959) (Pl. 24, Fig. 4, PP. 103)

Collection Number and Date: HP/MAN/4b, $20 / 5 / 2004$

Locality: Nalsar Pond, Mandi.

Description: Cells without sheath $8 \mu \mathrm{m}$ in diameter with sheath $10 \mu \mathrm{m}$ in diameter.

3. C. tenax (Kirchn.) Hieron (Pl. 1, Fig. 6) Desikachary, T.V. (1959) (Pl. 26, Fig. 7, 16, PP. 103)

Collection Number and Date: HP/UNA/4c, 4/4/2006

Locality: Saloh Ghaluwal Pond, Una.

Description: Cells without sheath $19 \mu \mathrm{m}$ in diameter with sheath $29 \mu \mathrm{m}$ in diameter.

Genus: Aphanocapsa Nag.

1. Aphanocapsa pulchra (Kutz.) Rabenh (Pl. 1, Fig. 8) Desikachary, T.V. (1959) (Pl. 21, Fig. 2, PP. 132)

Collection Number and Date: HP/UNA/4c, $4 / 4 / 2006$

Locality: Saloh Ghaluwal Pond, Una. 
Description: Cells $3.5 \mu \mathrm{m}$ in diameter.

Genus: Aphanothece Nageli

1. Aphanothece castagnei (Breb.) Rabenh (Pl. 1, Fig. 9) Desikachary, T.V. (1959) (Pl. 21, Fig. 8, PP. 140)

Collection Number and Date: HP/UNA/4c, 4/4/2006

Locality: Saloh Ghaluwal Pond, Una.

Description: Cells $6 \mu \mathrm{m}$ in length; $3.5 \mu \mathrm{m}$ in width

Genus: Merismopedia Meyen

1. Merismopedia glauca (Ehr.) Nag. (Pl. 1, Fig. 10) Desikachary, T.V. (1959) (Pl. 29, Fig. 5; PP. 155) Prasad, B.N. and Srivastava, M.N. (1992) (Pl. 6 Fig. 5, PP. 44)

Collection Number and Date: HP/MAN/2b, $19 / 5 / 2004$

Locality: Jarol Khad, Mandi.

Description: Diameter of cell 3-4 $\mu \mathrm{m}$, diameter of colony $10 \mu \mathrm{m}$.

Genus: Coelosphaerium Nag.

1. Coelosphaerium kützingianum Nag. (P1. 1, Fig. 11) Desikachary, T.V. (1959) (P1. 28, Fig. 7, 8, PP. 148)

Collection Number and Date: HP/UNA/4c, $4 / 4 / 2006$

Locality: Saloh Ghaluwal Pond, Una.

Description: Cells $2.5-6 \mu \mathrm{m}$ in diameter, colony $110 \mu \mathrm{m}$ in diameter.

Genus: Gomphosphaeria Kütz.

1. Gomphosphaeria aponina Kütz. (P1. 1, Fig. 12) Desikachary, T.V. (1959) (Pl. 28, Figs. 1-3, PP. 150)

Collection Number and Date: HP/SIM/I 5b, $25 / 5 / 2004$

Locality: Kufri Pond, Shimla.

Description: Cells $6 \mu \mathrm{m}$ in length; $4 \mu \mathrm{m}$ in width.

Order - Chamaesiphonales
Family: Dermocarpaceae

Genus: Stichosiphon Geitler

1. Stichosiphon sansibaricus (Hieron.) Drouet et Daily (Pl. 1, Fig. 13) Desikachary, T.V. (1959) (P1. 32, Fig. 9-13, PP. 176)

Collection Number and Date: HP/HAM/12c, 4/6/2005

Locality: Kunah Khad Dindwi, Hamirpur.

Description: Cells 4-5 $\mu \mathrm{m}$ in width.

Order - Nostocales

Family: Oscillatoriaceae

Genus: Oscillatoria Vaucher

1. Oscillatoria perornata Skuja (P1. 2, Fig. 15) Desikachary, T.V. (1959) (Pl. 41, Fig. 14, PP. 205)

Collection Number and Date: HP/HAM/I Ic, 4/6/2005

Locality: Jhanyari Khad Bhota, Hamirpur.

Description: Trichome $10 \mu \mathrm{m}$ broad, 3-4 $\mu \mathrm{m}$ long.

2. O. princeps Vaucher ex Gomont (Pl. 2, Fig. 17) Desikachary, T.V. (1959) (Pl. 37, Fig. 11, PP. 210)

Collection Number and Date: HP/MAN/4b, 20/5/2004

Locality: Nalsar Pond, Mandi.

Description: Trichome $45 \mu \mathrm{m}$ broad, $5 \mu \mathrm{m}$ long.

3. O. sancta (Kütz.) Gomont (Pl. 1, Fig. 14) Desikachary, T.V. (1959) (Pl. 42, Fig. 10, PP. 203).

Collection Number and Date: HP/MAN/4b, 20/5/2004

Locality: Nalsar Pond, Mandi.

Description: Trichome $2.5 \mu \mathrm{m}$ long, $11 \mu \mathrm{m}$ broad

4. O. terebriformis Ag. ex Gomont (P1. 1, Fig. 16) Desikachary, T.V. (1959) (Pl. 38, Fig. 16, PP. 217) Collection Number and Date: HP/UNA/4c, 4/4/2006

Locality: Saloh Ghaluwal Pond, Una.

Description: Trichome $3 \mu \mathrm{m}$ long, $6 \mu \mathrm{m}$ broad. 
Genus: Lyngbya Agardh

1. Lyngbya hirgei Smith, G.M. (Pl. 1, Fig. 20) Desikachary, T.V. (1959) (Pl. 50, Fig. 7, PP. 296)

Collection Number and Date: $\mathrm{HP} / \mathrm{MAN} / 4 \mathrm{~b}$, 20/5/2004

Locality: Nalsar Pond, Mandi.

2. L. martensiana Menegh. ex Gomont (Pl. 2, Fig

1) Desikachary, T.V. (1959) (Pl. 52 Fig. 6, PP. 318)

Collection Number and Date: HP/MAN/4b, 20/5/2004

Locality: Nalsar Pond, Mandi.

Description: Trichome $11 \mu \mathrm{m}$ in width; $1.5 \mu \mathrm{m}$ in length.

3. L. major Menengh. ex Gomont (P1. 1, Fig. 19)

Desikachary, T.V. (1959) (Pl. 52, Fig. 11, PP. 320)

Collection Number and Date: HP/MAN/4b, 20/5/2004

Locality: Nalsar Pond, Mandi.

4. L. putealis Mont. ex Gomont (Pl. 1, Fig. 18) Desikachary, T.V. (1959) (P1. 52, Fig. 12, PP. 317)

Collection Number and Date: HP/MAN/4b, 20/5/2004

Locality: Nalsar Pond, Mandi.

Description: Filament $16 \mu \mathrm{m}$ in width, trichome $12-13 \mu \mathrm{m}$ in width and $4-5 \mu \mathrm{m}$ in length.

Family: Nostocaceae

Genus: Anabaenopsis (Wolosz.) Miller sensu strict

1. Anabaenopsis circularis (G.S. West) Wolosz. et Miller var. javanica Wolosz. (Pl. 2, Fig. 2) Desikachary, T.V. (1959), (Pl. 63, Fig. 3 and 5, PP. 354)

Collection Number and Date: $\mathrm{HP} / \mathrm{HAM} / 5 \mathrm{c}$, $2 / 6 / 2005$

Locality: Taal Pond, Hamirpur.

Description: Cells 4-5 $\mu \mathrm{m}$ broad; heterocyst $\mu \mathrm{m}$ broad
Genus: Cylindrospermum Kutzing

1. Cylindrospermum stagnate (Kutz.) Born et and Flah. (Pl. 2, Fig. 3) Desikachary, T.V. (1959), (P1. 65, Fig. 9, PP. 363)

Collection Number and Date: HP/SIR/11a, $8 / 11 / 2003$

Locality: Giri river Yashawantnagar, Sirmaur.

Description: Cells 3-4 $\mu \mathrm{m}$ broad; heterocyst $5 \mu \mathrm{m}$ broad, akinete $8 \mu \mathrm{m}$ broad.

Genus: Nostoc Vaucher

1. Nostoc carneum Ag. ex Born. et Flah. (Pl. 2, Fig. 5) Desikachary, T.V. (1959), (Pl. 69, Fig. 6, PP. 381)

Collection Number and Date: HP/MAN/6c, 6/4/2006

Locality: Suketi Khad Ner Chowk, Mandi.

Description: Cells $3 \mu \mathrm{m}$ broad; heterocyst $4 \mu \mathrm{m}$ broad.

2. N. muscorum Ag. ex Born. et Flah. (Pl. 2, Fig.

4) Desikachary, T.V. (1959) (Pl. 70, Fig. 2, PP. 385)

Collection Number and Date: HP/MAN/6c, 6/4/2006

Locality: Suketi Khad Ner Chowk, Mandi.

3. $N$. passerifiianum (De. Not.) Bornet ex Born. et Flah. (Pl. 2, Fig. 6) Desikachary, T.V. (1959), (P1. 69, Fig. 4, PP. 385)

Collection Number and Date: HP/MAN/4b, 20/5/2004

Locality: Nalsar Pond, Mandi.

Description: Cells 3-4 $\mu \mathrm{m}$ broad; heterocyst $5 \mu \mathrm{m}$ broad.

Genus: Anabaena Bory

1. Anabaena circmalis Rabenhorst ex Born. et Flah. var. crassa Ghose (Pl. 2, Fig. 8) Desikachary, T.V. (1959), (Pl. 77, Fig. 5, PP. 414)

Collection Number and Date: HP/HAM/5c, $2 / 6 / 2005$ 
Locality: Taal Pond, Hamirpur.

Description: Cells 3-4 $\mu \mathrm{m}$ broad; heterocyst $5 \mu \mathrm{m}$ broad.

2. A. macrospora Klebahm (Pl. 2, Fig. 11) Prescott, G. W. (1951) (Figs. 4-6, Pl. 117, PP. 517)

Collection Number and Date: HP/MAN/Sc, $6 / 4 / 2006$

Locality: Nalsar Pond, Mandi.

Description: Cells $7 \mu \mathrm{m}$ in width, heterocyst $7 \mu \mathrm{m}$ in width.

3. A. fertilissima Rao, C. B. (Pl. 2, Fig. 9) Desikachary, T.V. (1959), (Pl. 74, Fig. 1, PP. 398)

Collection Number and Date: HP/UNA/4c, $4 / 4 / 2006$

Locality: Saloh Ghaluwal Pond, Una.

Description: Cells 5-6 $\mu \mathrm{m}$ broad; heterocyst $9 \mu \mathrm{m}$ broad.

4. A. laxa (Rabehn.) (Pl. 2, Fig. 10) Desikachary, T.V. (1959), (, PP. 413)

Collection Number and Date: HP/UNA/4c, $4 / 4 / 2006$

Locality: Saloh Ghaluwal Pond, Una.

Description: Cells $4 \mu \mathrm{m}$ broad; heterocyst $5 \mu \mathrm{m}$ broad and $7 \mu \mathrm{m}$ long.

5. A. helicoidea Bernard (Pl. 2, Fig. 12) Prescott, G. W. (1951) (Fig. 8, Pl. 116, PP. 515)

Collection Number and Date: $\mathrm{HP} / \mathrm{HAM} / 5 \mathrm{c}$, $2 / 6 / 2005$

Locality: Taal Pond, Hamirpur.

Description: Cells $5 \mu \mathrm{m}$ broad.

6. A. torulosa (Carm.) Lagerh. (P1. 2, Fig. 7) Desikachary, T.V. (1959), (Pl. 71, Fig. 6, PP. 415)

Collection Number and Date: HP/MAN/5c, $6 / 4 / 2006$

Locality: Nalsar Pond, Mandi.

Genus: Pseudanabaena Lauterborn
1. Pseudanabaena schmidlei Jaag. O. (Pl. 2, Fig.

13) Desikachary, T.V. (1959), (Pl. 75, Fig. 4, PP. 419)

Collection Number and Date: HP/SOL/10a, $8 / 11 / 2003$

Locality: Mohan Baodi Dharmapur, Solan.

Description: Cells $10 \mu \mathrm{m}$ broad $9 \mu \mathrm{m}$ long.

Sub Family: Aulosirae

Genus: Nodularia Mertens

1. Nodularia spumigena Mertens ex Born. et Flah. (Pl. 2, Fig. 14) Desikachary, T.V. (1959), (Pl. 80, Figs. 13, 14, PP. 423)

Collection Number and Date: HP/UNA/4c, 4/4/2006

Locality: Saloh Ghaluwal Pond, Una.

Description: Cells $7 \mu \mathrm{m}$ broad; heterocyst $10 \mu \mathrm{m}$ broad.

Family: Microchaetaceae

Genus: Fortiea De Toni, J.

1. Fortiea bossei (Fremy) comb. nov. (P1. 2, Fig. 15) Desikachary, T.V. (1959), (Pl. 82, Fig. 13-15, PP. 516)

Collection Number and Date: $\mathrm{HP} / \mathrm{MAN} / 5 \mathrm{c}$, $6 / 4 / 2006$

Locality: Nalsar Pond, Mandi.

Family: Rivulariaceae

Genus: Calothrix Ag.

1. Calothrix braunii (A. Br.) Bornet et Flahault (Pl. 2, Fig. 16) Desikachary, T.V. (1959), (Pl. 114, Fig. 3, PP. 535)

Collection Number and Date: $\mathrm{HP} / \mathrm{UNA} / 4 \mathrm{c}$, $4 / 4 / 2006$

Locality: Saloh Ghaluwal Pond, Una.

2. C. brevissima West G.S. var. moniliforme Ghose (Pl. 2, Fig. 17) Desikachary, T.V. (1959) (Pl. 112, Fig. 8, PP. 533)

Collection Number and Date: HP/UNA/4c, $4 / 4 / 2006$

Locality: Saloh Ghaluwal Pond, Una.

Description: Cells 4-8 $\mu \mathrm{m}$ broad; heterocyst $9 \mu \mathrm{m}$ broad. 

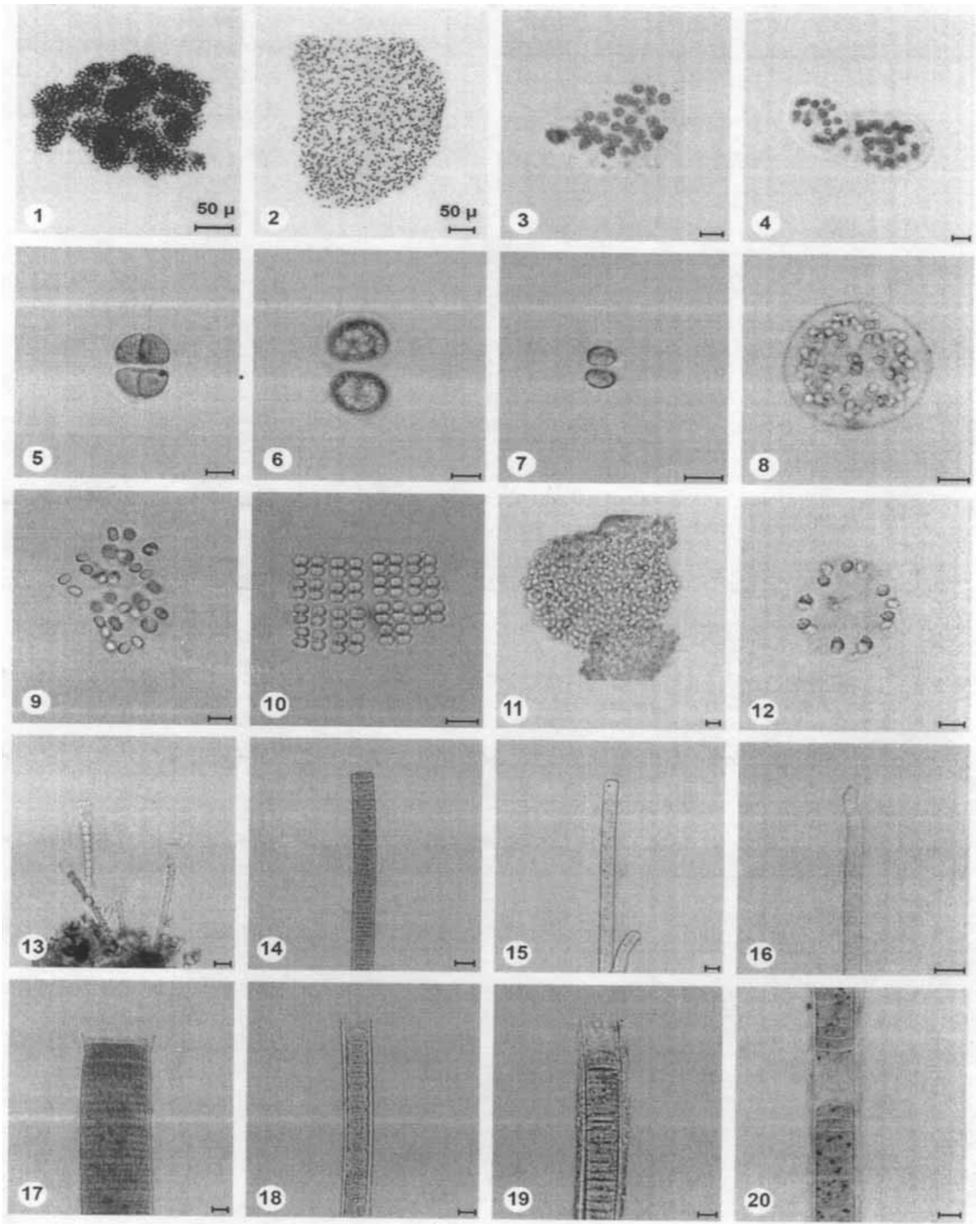

Plate 1. Fig. 1. Microcystis aeruginosa Kutzing, Fig. 2. M. flos-aquae (Wittr.) Kirchner, Fig. 3. *M robusta (dark) Nygaard, Fig. 4. M. lamelliformis Holsinger, Fig. 5. Chroococcus limnelicus Lemmermann, Fig. 6. * C. tenax (Kirchn.) Hieron, Fig. 7. C. minutus (Kutz.) Nageli, Fig. 8. *Aphanocapsa pulchra (Kutz.) Rabenhorst, Fig. 9. Aphanothece castagnei (Breb.) Rabenhorst, Fig. 10. Merismopedia glauca (Ehr.) Nageli, Fig. 11. Coelosphaerium Kützingianum Nageli, Fig. 12. Gomphosphaeria aponina Kützing, Fig. 13. *Stichosiphon sansibaricus (Hieron.) Drouet and Daily, Fig. 14. Oscillatoria sancta (Kütz.) Gomont, Fig. 15. *O. peronata Skuja f. attenuata Skuja, Fig. 16. O. terebriformis Ag. ex Gomont, Fig. 17. *O. princeps Vaucher ex Gomont var. crassa Rao, Fig. 18. *Lyngbya putealis Mont. ex Gomont, Fig. 19. L. major Menenghini ex Gomont, Fig. 20. L. birgei Smith 

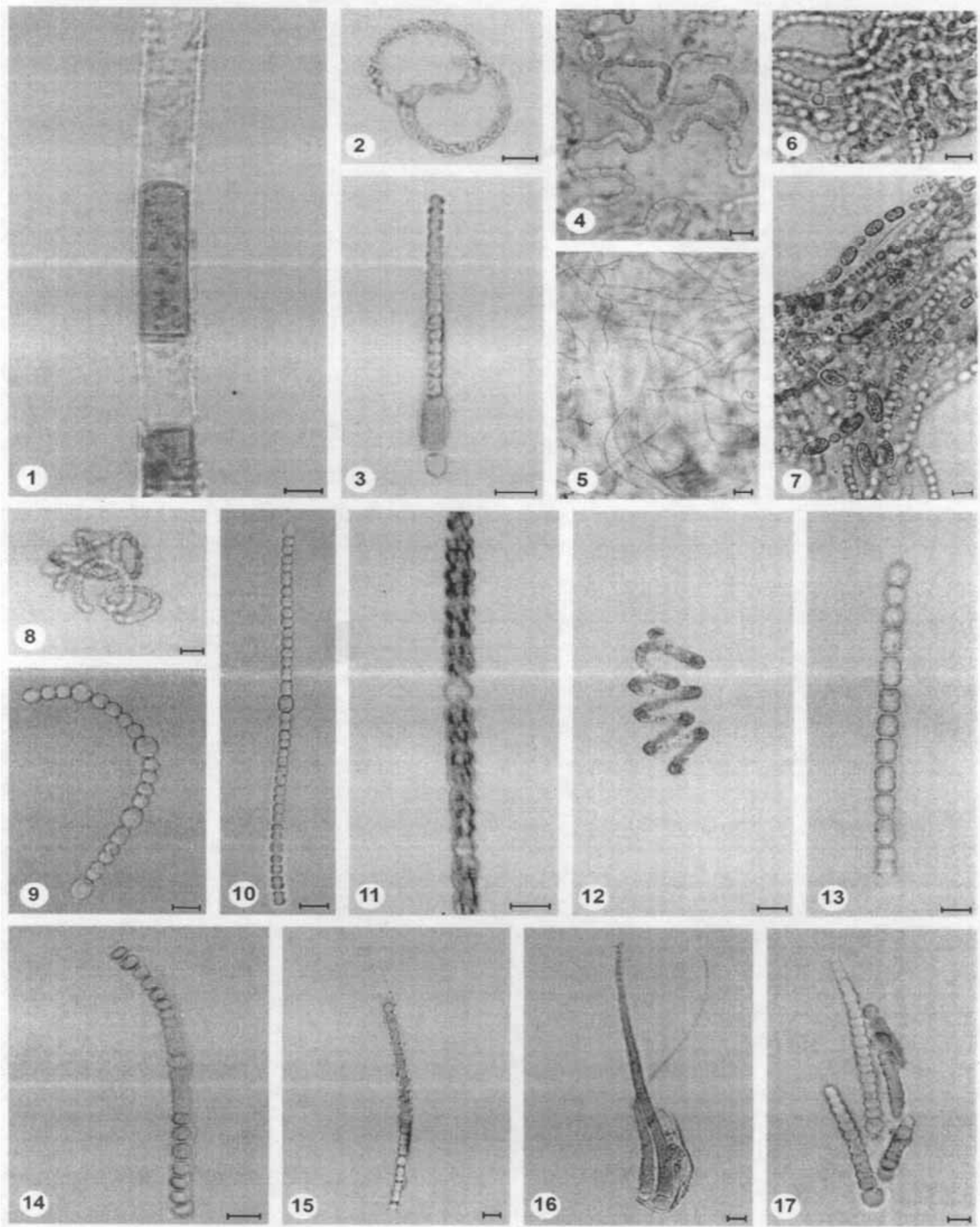

Plate 2. , Fig. 1. Lyngbya martensiana Menegh. ex Gomont, Fig. 2. Anabaenopsis circularis (G.S. West) Wolosz. and Miller var. javamca Wolosz., Fig. 3. Cylindrospermum stagnate (Kutz.) Bornet and Flahault, Fig. 4. Nostoc muscorum Ag. ex Born. and Flah, Fig. 5. N. carneum Ag. ex Born. and Flah., Fig. 6. N. passerinianum (De. Not.) Bomet ex Born. and Flah, Fig. 7. Anabaena torulosa (Carm.) Lagerheim, Fig. 8. A. circinalis Rabenhorst ex Born. and Flah var. crassa Ghose, Fig. 9. *A. fertilissima Rao, Fig. 10. *A. laxa (Rabehn.) Brown, Fig. 11. A. macrospora Klebahm., Fig. 12.*A. helicoidea Bernard, Fig. 13. Pseudanabaena schmidtei Jaag, Fig. 14. Nodularia spumigena Mertens ex Born. and Flah, Fig. 15. Fortiea bossei (Fremy) comb. Desikachary, Fig. 16. Calothrix braunii (Br.) Bornet and Flahault, Fig. 17. C. brevissima West var. momliforme Ghose. 


\section{ACKNOWLEDGEMENT}

Authors are thankful to Ministry of Environment and Forest, New Delhi for financial assistance and to the Head, Department of Botany, University of Lucknow, Lucknow for facilities. R.K. Dwivedi and C.P. Shukla (NET-S.R.F.) are thankful to C.S.I.R., New Delhi, for financial assistance.

\section{REFERENCES}

Darbal, P.K., A.M. Bendre and L. Singh. 1978. Cyanophyceae of Rishikesh and its adjacent foothills. Botanica Progress 1:41-44.

Desikachary, T.V. 1959. Cyanophyta, A Monograph. I.C.A.R., New Delhi.

Gupta, A.B. and G.U. Nair. 1962. A contribution to the algal-flora of Garhwal. J. Agra Univ. Res. Sci. 11(3):227-240.

Habib, I. 1999. Desmids of Shimla, Himanchal Pradseh. Acta Botanica 24(1):119.

Habib, I. and U.K. Chaturvedi. 1999. A systematic account of Chlorococcales from Ramnagar, Kumaon Himalaya. Phycos 38(1\&2):97-100.

Habib, I. and U.K. Chaturvedi. 2000. A systematic account of Chlorococcales from Kumaon Himalaya. Phycos 39(1\&2): 113-118.

Habib, I. and U.K. Chaturvedi. 2001. Epiphytic algal flora of Kumaon Himalayas: Chaetophorales. Phycos 40(1\&2):79-81.

Habib. I., J.O. Ghildiyal, C. Negi and U.K. Chaturvedi. 1998. A systematic account of Chlorococcales from Kotdwar. Phycos 37(1\&2): 125-129.

Kamat, N.D. 1968. Algae of Shimla (Miscellaneous Note). J. Bombay Nat. Hist. Soc. 65(1):271-277.
Kamat, N.D. 1973. Algae of Nainital. J. Bombay Nat. Hist. Soc. 70(3):582-586.

Khan, M. 1970. Algal flora of Dehradun-I, Myxophyceae. Phycos 9(2):126-131.

Misra, P.K., R.K. Dwivedi and C.P. Shukla. 2006. Some fresh-water desmids from District Mandi, Himachal Pradesh. Phytotaxonomy 6:120-125.

Prasad, B.N. and M.N. Srivastava. 1992. Fresh Water Algal Flora of Andaman and Nicobar Islands. Vol. 1. B. Singh, Dehradun, India, $369 \mathrm{pp}$.

Prescott, G.W. 1951. Algae of the western great lakes area. W.M.C. Brown Publishers, Dubuque, Iowa, USA, 977 pp.

Seth, K.P., M.K. Seth and P.K. Misra. 2006. A review of literature on algal flora of Himachal Pradesh. Phytotaxonomy 5:35-57.

Shukla, S.K. and P.K. Misra. 2006. Dimorphococcus prasadii sp. nov. (Chlorophyceae, Chlorococcales) from foothills of Western Himalayas, India. Crypt. Algol. (accepted).

Shukla, S.K., P.K, Misra and C.P. Shukla. 2007. Chlorococcalean algae from the foothills of Indo-Westem Himalaya. J. Indian Bot. Soc. (accepted).

Singh, K.P. 1959. Myxophyceae of Kumaon Hills, U.P., India-1. Proceedings of Indian Acad. Sci. 49:161-166.

Singh, K.P. 1961. Myxophyceae of Kumaon Hills, U.P., India-11. Uttar Bharti 8(1):101-108.

Talpasayi, E.R.S. 1962. The myxophyceae of the Kumaon Hills, U.P., India-11. Proc. Indian Acad. Sci. 55:251-255. 